$\begin{array}{ll}\text { Italique } & \text { Italique } \\ \text { Poésie italienne de la Renaissance }\end{array}$

IV | 2001

Varia

\title{
Contro la luna. Appunti sul motivo antilunare nella lirica d'amore da Serafino Aquilano al Marino
}

\section{Roberto Gigliucci}

\section{(2) OpenEdition \\ Journals}

Edizione digitale

URL: http://journals.openedition.org/italique/159

DOI: 10.4000/italique.159

ISSN: 1663-4438

\section{Editore}

Librairie Droz

\section{Edizione cartacea}

Data di pubblicazione: 1 dicembre 2001

Paginazione: 19-29

ISBN: 2-600-00826-1

ISSN: 1423-3983

Notizia bibliografica digitale

Roberto Gigliucci, « Contro la luna. Appunti sul motivo antilunare nella lirica d'amore da Serafino

Aquilano al Marino », Italique [Online], IV | 2001, online dal 06 octobre 2009, consultato il 01 mai 2019. URL : http://journals.openedition.org/italique/159; DOI : 10.4000/italique.159 
R овеR T O GIGLIU C C I

C O N TR O LA L U A.

A P P UNTI S UL MOTIVOANTILUNARE

NELLA LIR I C A D’ A M ORE

DA SERAFINO A Q ILANOAL MARINO 


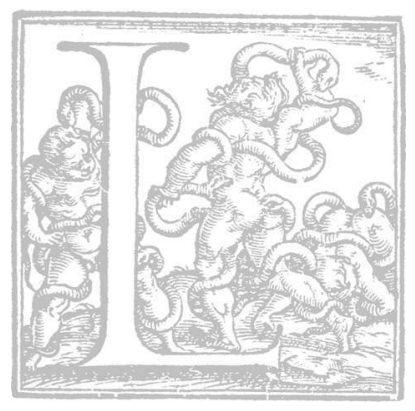

A posizione del poeta contro la Luna, di invettiva e accusa, è minoritaria rispetto alle infinite occorrenze dell'allocuzione alla luna vaga e coronata di epiteti positivi (argentea, bella, candida, chiara, incantata, pietosa ecc.). Una soluzione intermedia evidenzia le caratteristiche piu "criticabili" della luna, come l'instabilità o il gelo, senza scegliere però decisamente l'atteggiamento denigratorio misoselenico (allora la luna è fredda, gelata, incostante, instabile ecc.). Nella stampa delle Rime del I598, Guarini include un madrigale (Splende la fredda luna, LI rubrica: "Fredda bellezza») $)^{\mathrm{I}}$ il cui concetto è basato sul parallelo luna-madonna: entrambe rimangono gelide, pur subendo il caldo splendore del sole reale la prima e di quello metaforico-amoroso la seconda. Si tratta di una variazione arguta sul motivo topico della freddezza della luna, un esempio di quell'atteggiamento poetico relativo alla luna che non perviene sino all'invettiva, ma seleziona attributi in qualche modo negativi della luna, costruendovi sopra, come in questo caso, un ricamo epigrammatico.

Il topos dell'invettiva in Lunam è ampiamente sviluppato da Serafino Aquilano ${ }^{2}$ nel capitolo Inuida Luna, instabile et proterua. ${ }^{3}$ La coppia aggettivale di destra è petrarchesca: cfr. Rvf. 319, 5 «Misero mondo, instabile et protervo»; il dato dellinvidia lo ritroveremo giù giù fino a Marino: "Oh luna, invida luna», La sampogna VIII, I295; non si dimentichi poi l'attacco del celebre sonetto anticortigiano dell'Aquilano. Gli attributi della luna offerti copiosamente da Serafino sono canonici e avranno ampia fortuna nel filone antilunare: assenza di luce autonoma, freddezza, instabilità (ribadita al v. Iб) e cosi via. La climax serafiniana culmina (momentaneamente, poiché la gradazione dell'invettiva riprende più avanti fino alla fine) con l'eccesso di definire la luna «un nocturno spion senza mercede» (I8), modulazione iperbolica su un'immagine non inconsueta, quella dell'astro argenteo che spia gli amanti (c'è anche in un epigramma della Palatina, V, I23, però assente dalla Planudea). ${ }^{4}$ Il motivo della scoperta dei furta di Marte e Venere (25 ss.) è in genere attribuito al Sole, come in Ovidio Met. IV, I69 ss. e in Lorenzo, Furtum Veneris et Martis. Così come predicato del sole è generalmente lo scorno per la vittoria dello splendore di madonna su di lui; nel capitolo di Serafino è la Luna a dover temere il confronto con la tanto più chiara luminosità della «sua dea», di madonna, spostando un motivo squisitamente eliofobico (da Petrarca a De Jennaro a Britonio ${ }^{7}$ ) sul piano selenofobico. Si ripropone, quindi, nella tradizione lirica un parallelismo tra momento eliolatrico (si pensi soltanto a Vittoria Colonna) vs eliofobico (da Britonio, anzi, già da Petrarca) e momento selenolatrico (mag- 
gioritario, culminante in un Cariteo) vs selenofobico, o misoselenico che dir si voglia.

Qualche vaga coincidenza col capitolo di Serafino presenta il capitolo ariosteo $\mathrm{O}$ nei miei danni più che 'l giorno chiara ( $I X$, in dittico - speculare - col precedente, "properiaiano" cap. VIII), probabilmente del I5I3. Rivolgendosi alla luna, dopo due terzine di allocuzione alla notte, il poeta esordisce "Tu che di si gran luce altiera vaì (7), e si può allora evocare il verso serafiniano «Ingrata al sol, per cui vai tanto altera» (22); ancora, cfr. Ariosto «oh quanta occasione ora mi vieti» (29) e Serafino «Ab! quanto ogni mortal di te si duole!». ${ }^{8}$ L'elemento, come vedremo, ricorrente della luminosità riflessa della luna era espresso da Serafino in una intera terzina ("Luce non hai, se non quanto che'l sole / talhor per sua virtù digna mirarte, / quando dal nostro ciel partir si vole», 4-6: «Phoebe [...] lumine non suo», Seneca, Medea 97); Ariosto lo riassume in un sintagma, «il tuo bastardo lume» (32) che è calco del latino notho lumine, presente in Lucrezio (V, 575) e Catullo (34, I5-I6). Il richiamo a Endimione, che curiosamente nell'A quilano mancava, in Ariosto è presente, e con tutta la sensualità tipica del poeta ferrarese («quando in braccio al pastor nuda scendesti»); tuttavia il mito endimioneo sembrerebbe contaminato con quello relativo all' amore di Pan e Diana: infatti i versi ib ss. del capitolo dell'Ariosto ("ché non amor, ma la mercé d'un vello, / che di candida lana egli t'offerse, / lo fe' parer alli occhi tuoi si bello») sono riferiti a Endimione, ma il dono seduttivo della bianca lana è in realtà opera di Pan, giusta anche lo stesso Virgilio: "Munere sic niveo lanae, si credere dignum est, / Pan deus Arcadiae captam te, Luna, fefellit / in nemora alta vocans; nec tu aspernata vocantem» (Georg. III, 39I-93; cfr. Macrobio, Sat. conv. V, 22, 9). ${ }^{9}$ In un sonetto attribuito dal Raffaelli all'Alamanni, D'angosciosi pensier già lasso e stanco, abbiamo ancora una invida, fredda Luna che risveglia il poeta dal sogno d'amore. ${ }^{\circ}$

Nell'egloga vi di Girolamo Muzioi ${ }^{11}$ la bianca fredda casta Luna è accusata di infedeltà a Endimione, in quanto ha intrecciato relazione con Pan in Arcadia (cfr. il brano virgiliano delle Georgiche, sopra citato). Il tradimento e il dolore di Endimione ha qualcosa di tragico, ma anche di grottescosatirico.

Dedicato alla Luna crudel è un madrigale della raccolta di Girolamo Parabosco, pubblicata nel I5sI. ${ }^{\mathrm{I} 2}$ La situazione è particolare: la luna sembra l'oggetto dell' amore disperato del poeta, come nel caso del Cariteo; la luna nega $i$ suoi raggi luminosi al poeta, lasciandolo sepolto nella tenebra notturna. Allora scatta la reazione di invettiva, pressoché estranea all'universo amoroso cortese e petrarchesco, ma non a quello classico: il poeta si augura che il sole non conceda più luminosità alla luna, che le «nubi importune» la ricoprano (cfr. Rvf. 66, I «l'importuna nebbia» e quindi Sannazaro LXXXIII, 30 «Poi 
che importuna nube il sol mi cela»), finché essa arrivi ad essere odiata da tutto il mondo, compreso Endimione (chiusa iperbolico-paradossale: «da te fugga Endimione ancora»).

Torquato Tasso scrive una canzone intera "Contro la luna la quale aveva interrotto un suo viaggio notturno», testo che il Solerti pubblica nel libro terzo della sua edizione, fra rime amorose estravaganti in parte riconducibili al periodo dei canzonieri per la Bendidio e la Peperara (n. 383$) ;{ }^{13}$ una redazione antecedente è testimoniata dal ms. Chigiano. ${ }^{14}$ L'attacco è fortemente classicheggiante e aggressivo; il poeta rifiuta la lira per un «altro istrumento», più mordace, satirico, a disonorare «l'iniqua Luna». Seguono due stanze in cui si descrive l'evento: la luna ha illuminato improvvisamente la notte, mentre il poeta tentava di raggiungere furtivamente il luogo dove era l'amata. La stanza seguente si scaglia direttamente contro la "Luna rubella / d'ogni pietà», definita «invidiosa» dei piaceri amorosi e «tenebrosa» in sé, poiché la luce gli viene data dal Sole. Le stanze ulteriori affrontano esempi mitologici: prima di tutti l'episodio dell'amore per Endimione, che contraddice alla fredda castità dell'iconografia tradizionale, poi i concubiti con Pan e l'evocazione del dono della lana, già anche nel Muzio e in Ariosto. Si chiama in causa poi Orione, il possente e bellissimo cacciatore, amato dall'Aurora: Tasso segue la versione del mito per cui Diana si invaghi anch'essa di Orione e Apollo, intimorito, fece si che Diana lo uccidesse per sbaglio. Tasso descrive il pianto di Diana sul corpo esanime di Orione, facendole recitare un breve monologo tragico, tutto tramato di variazioni sul motivo ossimorico della vita-morte: «Ah man, voi pronte / a l'altrui morte, vita a me togliesti! / Ché non si può chiamare / vita or la mia, / se non vogliam dir viva / chi de l'alma e del cor il fato ha privas (73-77). Questa particolare redazione del mitologema orioneo si trova ad esempio in Igino, non nelle Fabulae, ma nel De astronomia (II, 34), " che potrebbe essere fonte per Tasso. La penultima stanza della canzone evoca il mito celebre di Atteone, trasformandone maliziosamente la dinamica: Diana avrebbe fatto sbranare Atteone trasformato in cervo perché rifiutata da lui, come Fedra con Ippolito, per intenderci: "Ver è, se ben si tacque, / ch'egli a forza e con voglia aspra e severa / da le tue braccia sciolto / se 'n gisse, mentre tu d'ardor ripiena / al collo gli facei stretta catena) (83-88).

Arriviamo a Giovan Battista Marino, attraverso un percorso che, come si ̀̀ visto, dalla lirica cortigiana giunge alla lirica manierista e attraverso questa alla civiltà primo-barocca, al Marino e al marinismo (linea non priva di coerenza, come ci indica, fra gli altri, il Rossi a conclusione del suo saggio su Serafino Aquilano). ${ }^{16}$ Nelle Amorose delle Rime I602, Marino colloca un dittico $^{17}$ costituito da un sonetto di preghiera alla Luna e da un secondo di invettiva. L'invocazione del primo si apre con un'ottativa riccamente classicheggiante, cui segue la supplica alla Luna affinché non illumini l'oscurità, 
permettendo al poeta di consumare $i$ suoi «furtivi amori». Le «perle di gelo» che la luna fa piovere sulla terra rimandano a preziosità tutte tassiane (Rime 237, 5-6, ma col precedente di Rainerio, Cento sonetti n. I8, Era il mar cheto: et l'alte Selve e i Prati: «perle d'un vivo trasparente gielo» 6; il son. descrive l'aurora sconfitta dal più bel sole di madonna, secondo il modulo consueto); ${ }^{18}$ naturalmente anche il Cariteo viene evocato nel commento, a proposito dell'elefante, «indica fera», che adora purificato la luna. La citazione obbligata dell'amore per Endimione, poi, è realizzata da Marino con l'inserzione squisita di un doppio ossimoro: "s'è ver ch'errante / accesa il freddo sen di dolce arsura / gisti di Latmo infra l'ombrose piante» (9-II). La chiusa concettosa gioca sullo splendore notturno lunare e quello dell'incendio amoroso. Il sonetto seguente, post eventum, si scaglia contro la Luna che non ha favorito il poeta: esordisce con la negazione dei tradizionali epiteti elogiativi (Né pietosa, né lucente, né pura, né gentil, né bella) sostituiti con gli attributi perversa, sorda, rigida, algente. La giusta sede della cornuta dèa è l'inferno; anche le macchie (lunari), che ne chiazzano il pallore, sono orrende. Mentre nel sonetto precedente si evocava la sognante storia d'amore con Endimione, qui si chiama in causa la relazione sensuale con Pan, torto Dio. Fino a concludere sul motivo magico del tessalo incanto («turbi il tuo lume ognor tessalo incanto»: si veda Orazio, Epod. 5, 45 «sidera excantata voce Thessala»), talché la dinamica di questo sonetto è quella discensiva verso il regno delle tenebre, dove Ecate sprofonda, e verso il regno magico-orrido, nonché nel dominio sessuale divino-ferino dionisiaco. I commentatori osservano come parte del materiale lessicale del dittico sia in comune con un altro sonetto delle Amorose, il 26, dedicato anch'esso «Alla Luna, per una donna chiamata Cinzia», tutto costruito sul parallelismo Luna-donna. Sono messi in gioco elementi positivi (splendore argenteo) ma soprattutto negativi: potere di vita e di morte, volubilità e freddezza ecc. La terzina finale scarta, indicando motivi di differenza fra la donna e la luna, secondo un modulo analogico con chiusa sorprendentemente oppositiva, pattern frequente nei sonetti comparativi di area meridionale (e non solo), come ha magistralmente messo in evidenza Raimondi. ${ }^{19}$ Coincidenze lessicali, peraltro inevitabili, si rilevano anche, esempigrazia, con un sonetto delle Boscherecce, ${ }^{2 \circ}$ dove però il poeta «priega la Luna che si dimostri», quindi la perorazione è speculare. Tornando invece al sonetto di parallelismo Lunadonna, mette conto ricordare almeno un precedente tardo-cinquecentesco, offerto da Erasmo di Valvasone, di cui si legga il sonetto seguente:

Notturna dea, tu con splendor che traggi dal fratello, e non tuo, rassembri bella; questa, che del tuo nome il mondo appella, 
la notte illustra e 'l dì co' propi raggi.

Tu con l'arco, tra pini ascosa e faggi, domi le fere cacciatrice snella; da questa movon tacite quadrella che pon far mansueti i cor selvaggi.

Te conducon duo cervi intorno il cielo;

più poeti alzan lei sopra le spere; tu nudri i corpi umani, ella gl'ingegni.

Ella invitta, tu vinta d'un bel velo.

Or mira s'a ragion contenda e spere da l'immortalità pregi più degni. ${ }^{21}$

L'anafora del tu rimanda a Marino, ma in Erasmo abbiamo un confronto differenziante, tutto a favore della donna (anch'essa di nome Cinzia) che supera ampiamente la casta dea lunare nella lode iperbolica del poeta. La luna è vinta e scornata, come il sole che soccombe in tante battaglie contro madonna aurora sorgente nella lirica di tradizione eliofobica.

$$
\text { ** }
$$

Nel secolo XVII si pubblicano due fondamentali repertori poetici "tematici", oggi riesumati, studiati e fatti fruttare dalla scuola svizzera che fa capo a Giovanni Pozzi e Ottavio Besomi: quello di G. Cisano, Tesoro di concetti poetici scelti da' più illustri poeti toscani e ridotti sotto capi per ordine d'alfabeto, Venezia, Deuchino e Pulciani, I6ro, e, più tardi, quello di G. B. Spada, Giardino degli epiteti, traslati et aggiunti poetici italiani, Bologna, V. Benacci, I665. Nel primo, alla voce Luna, troviamo una sezione In biasmo della luna (II parte pp. 64 ss.), dove c'è un canone già formato, un triumvirato: Ariosto, col capitolo, Tasso, con la canzone, Marino, col sonetto. Ed ecco la sequenza classicistica in Lunam, che ovviamente relega nella tenebrosa preistoria il testo dell' Aquilano e sorvola su quelli cinquecenteschi minori. Se andiamo invece alla voce Luna del grande volume dello Spada, tutto o quasi spostato su esempi barocchi, troviamo almeno due occorrenze della luna con epiteti offensivi: la perversa luna del sonetto mariniano, che conosciamo, e una maligna luna di Anton Giulio Brignole Sale. Ė iunctura di una terzina tratta dalle Instabilità dell'ingegno e precisamente da una epistola poetica che, nella terza giornata del prosimetro, si immagina scritta da Tancredi all'amata Clorinda: "Maligna Luna, ${ }^{22}$ a che spiegar quel lampo? / Ben de le fiere con ragion sei Dea, / s'invidij si d'un amator lo scampo» (7-9, p. 80 dell'edizione di Venezia, presso $i$ Giunti, I652). La robusta triade del Cisano costruiva un filone forte antilunare che andava dal primo classicismo cinquecentesco, ariosteo, passava 
per la via obbligata di Torquato Tasso e perveniva al trionfo del nuovo, pur nella tradizione, con Marino; questa potente dorsale si sfalda e si corrompe nel brulichio di epiteti, aggiunti, traslati dell'immenso libro-mondo offerto dallo Spada, dove inoltre una zona identificabile come antilunare non ̀̀ percepibile.

Anche un altro protagonista del primo Seicento, Tommaso Stigliani, scrive un sonetto contro la luna che, con l' importuno scoprirsi, ha rischiarato improvvisamente la notte, impedendo al poeta di compiere i suoi amorosi disegni (rubrica):

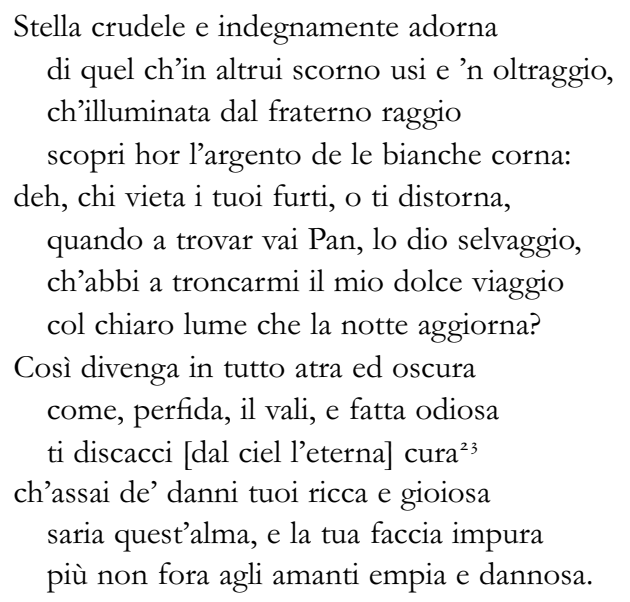

Questa è la lezione del sonetto secondo l'edizione Delle rime del signor Tomaso Stigliani. Parte prima, Venezia, G.B.Ciotti, del I6or. Nella stampa, assai ampliata e controllata, delle Rime del I605, ${ }^{24}$ il pezzo subisce notevoli variazioni, soprattutto nella seconda terzina, che introduce il motivo infernale: ${ }^{25}$

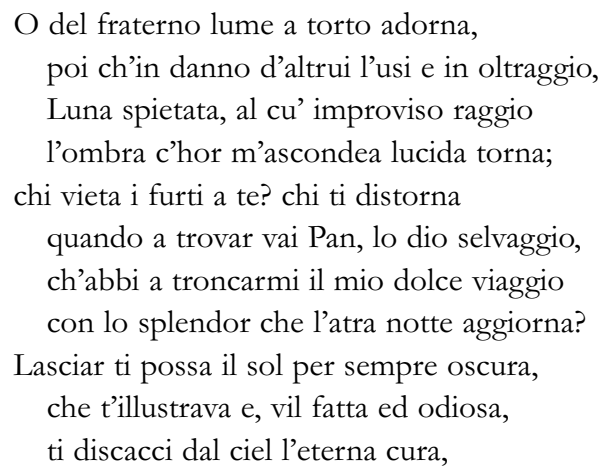


e tu resti al demon sotterra sposa

qual fosti ogn'hora, a ciò tua faccia impura

non sia più agli amanti empia e dannosa. ${ }^{26}$

Troviamo elementi già noti: la luce originata dal sole, la spietatezza (Luna spietata), gli amori con Pan. Le terzine augurano alla luna di restare per sempre orba di luce, di diventare odiosa agli dèi (cfr. Parabosco, madrigale Luna crudel, v. I3) e di precipitare agli inferi, dove del resto è la sua dimora come sposa del demon, dèa delle ombre (questa discesa è apparentabile con quella del sonetto mariniano). Curiosa una nota del Solerti, nella sua Vita di Torquato Tasso (I, Torino, Loescher, I895, pp. 783 sgg.). Tommaso Stigliani si fregiava di essere stato amico del Tasso in gioventì, ${ }^{27}$ in un dialogo manoscritto del sec. XVII, intitolato Le statue parlanti (per il Solerti introvabile, ma a lui segnalato da un amico erudito), il Tasso personaggio si querelerebbe del furto ai suoi danni da parte dello Stigliani, che gli avrebbe «rubata la mia canzone alla luna» $(p .786)$. In realtà, in questo filone antilunare, furtarelli e rubacchiamenti, o meglio, prelievi e intertesti sono rilevabili a più riprese e in più direzioni. Siamo cioè in presenza di una costellazione tematico-lessicale su un tema dato, insomma, in presenza di un tòpos. Che come tale andrà più a fondo studiato.

Roberto Gigliucci 


\section{Roberto Gigliucci}

Parte di questo intervento è stata materia di una relazione tenuta al convegno Serafino Aquilano nel quinto centenario della morte (I466-I500), svoltosi a L'Aquila fra il 3 e il s novembre del 2000.

i. Battista Guarini, Opere, a cura di M. Guglielminetti, Torino, UTET, i97i.

2. Naturalmente escludiamo da questo studio il settore comico-satirico-burlesco, anche se ad esempio certi modi serafiniani sono ovviamente spostati sul registro "realistico" o persino grottesco.

3. Die Strambotti des Serafino dall'Aquila. Studien und Texte zur italienischen Spiel- und Scherzdichtung des ausgebenden I5. Jabrbunderts, a cura di B. Bauer-Formiconi, München, W. Fink, 1967, p. 350 ss.

4. Anthologie grecque, première partie, Anthologie Palatine, tome II (livre V), a cura di P. Waltz e J. Guillon, Paris, Les Belles Lettres, 1960, pp. 63-64. Di una Luna indiscrète a proposito di Prop. I, 3, 3 I-33 parla S. Lunais, Les auteurs latins de la fin des Guerres Puniques à la fin du règne des Antonins [Recherches sur la Lune I.], Leiden, Brill, 1979, p. 295; si veda anche il paragrafo «La lune éclaire l'action», p. 283 ss., se pure senza frutti significativi nell'ottica della nostra ricerca.

5. Si vedano Rvf. 37, 8 I-82 «Le treccie d'òr che devrien fare il sole / d'invidia molta ir pieno»; Rvf. I 56, 5-6 «e vidi lagrimar que' duo bei lumi, / ch'àn fatto mille volte invidia al sole»; l'intero sonetto Rvf. i I 5 , ecc.

6. Sonetto Miracol grande nuovamente apparse, Serafino Aquilano, Rime e lettere, a cura di M. Corti, Bologna, Commissione per i testi di lingua, 1956, son. LIII, p. 95.

7. Britonio, Gelosia del sole, Venezia, Marchiò Sessa, i 53 I, passim; si vedano ad esempio i sonetti Essendo a rimirar Madonna i' volto o Quel per cui spesso dentro il cor m'adiro (si può ricorrere alla silloge La lirica rinascimentale, a cura di chi scrive, introduzione di J. Risset, Roma, Istituto Poligrafico e Zecca dello Stato, 2000, p. 443).

8. Stiamo citando il capitolo serafiniano dall'edizione Bologna I 503, cur. F. Flavio, cc. Giv-G3r. Per Ariosto ci atteniamo a Ludovico Ariosto, Rime a cura di S. Bianchi, Milano, Rizzoli, I992.

9. Anche Vincenzo Cartari mostrerà una certa confusione fra Endimione e Pan, in merito al dono lanoso: «E leggesi ancora che non per amore solamente fece la Luna copia di sé ad Endimione, overo Pan dio dell'Arcadia, come canta Virgilio, ma per avere da lui un gregge di belle pecore bianche»; cfr. l'edizione da cui cito, Imagini de $i$ dei de gli antichi, a c. di G. Auzzas, F. Martignago, M. Pastore Stocchi, P. Rigo, Vicenza, Neri Pozza, I996, p. 520 nota.

io. Versi e prose di Luigi Alamanni, a cura di P. Raffaelli, 2 voll., Firenze, Le Monnier, i 859 , I vol., p. 5; cfr. H. Hauvette, Un exilé fiorentin à la Cour de France an XVTème siècle: Luigi Alamanni, sa vie et son oewure, Paris, Hachette, 1903, p. 563: l'autore non trova il sonetto nei manoscritti consultati e mette in dubbio l'attribuzione.

II. Cfr. Le rime di Tullia di Aragona cortigiana del sec. XVI, a cura di E. Celani, Bologna, Commissione per i testi di lingua, 1969, p. 172 ss.

i 2. Girolamo Parabosco, Il primo libro dei madrigali, a cura di N. Longo, Roma, Bulzoni, I987, n. 56. 


\section{Contro la luna}

I 3. Cfr. anche Torquato Tasso, Opere, I, a cura di B. Maier, Milano, Rizzoli, I963, p. 445 ss.; Torquato Tasso, Le rime, t. I, a cura di B. Basile, Roma, Salerno, I 994, p. 344 ss.

I4. Torquato Tasso, Rime d'amore (secondo il cod. Chigiano L VIII 302), a cura di F. Gavazzeni, M. Leva, V. Martignone, Ferrara-Modena, ISR-Panini, I993, p. I 37 ss.

I 5. Hygin, L'astronomie, a cura di A. Le Boeuffle, Paris, Les Belles Lettres, I983, pp. 76-77.

I6. A. Rossi, Serafino Aquilano e la poesia cortigiana, Brescia, Morcelliana, I980.

i7. Giovan Battista Marino, Rime amorose, a c. di O. Besomi e A. Martini, FerraraModena, ISR-Panini, I 987 , p. I 35 ss.

I 8. Cito dall'edizione milanese dei Cento sonetti del I553, per Gio. A. Borgia, c. Bir.; cfr. G. Gorni, Un'ecatombe di rime. I «Cento sonetti» di Antonfrancesco Rainerio, «Versants», XV (1989), pp. 135-52.

19. E. Raimondi, Rinascimento inquieto, Torino, Einaudi, I994, p. 290.

20. Giovan Battista Marino, Rime boscherecce, a c. di J. Hauser-Jakubovicz, FerraraModena, ISR-Panini, I991, p. 59.

21. Il sonetto uscì a stampa nel I567, fra le Rime di diversi illustri autori. In lode della signora Cinzia Tiene Bracciadura (Padova, L. Pasquati); noi lo citiamo da Erasmo di Valvasone, Le rime, introduzione e note di G. Cerboni Baiardi, bibliografia e indici di A. del Zotto, Valvasone, Circolo Culturale Erasmo di Valvason, I993, p. 27.

22. Forse memoria del luce maligna di Aen. VI, 270 (Servio glossa obscura, Canali traduce avara luce).

23. Integro la lacuna con la lezione delle edizioni seguenti.

24. Cfr. O. Besomi, Ricerche intorno alla «Lira» di G. B. Marino, Padova, Antenore, I969, p. Iog.

25. Forse per influenza del Marino, di cui era uscita nel 1602 l'edizione delle Rime col son. antilunare sopra esaminato.

26. B. Croce, Lirici marinisti, Bari, Laterza, I9 10, pubblica il sonetto secondo una redazione ulteriormente ritoccata, tratta dall'edizione del 1625 , identica a quella del I623, ultima stampa, vivente l'autore, del Canzoniero ma non ultima fase redazionale dell'opera: cfr. O. Besomi, Esplorazioni secentesche, Padova, Antenore, I975, p. 55 s.; G. P. Maragoni, Stigliani «ne varietur». Appunti sulla riscrittura del «Polifemo», «Lettere italiane», I (1989), pp. 90-98, p. 93 s.

27. Cfr. O. Besomi, Ricerche cit., p. I Io nota 3. 